\title{
EFEITO DA PRESENÇA DE SEDIMENTO NA TOXICIDADE AGUDA DO SULFATO DE COBRE E DO TRICLORFON PARA TRÊS ESPÉCIES DE DAPHNIA
}

\author{
LUIS RICARDO ROMERO ARAUCO* \\ CLAUDINEI DA CRUZ" \\ JOAQUIM GONÇALVES MACHADO NETO***
}

\begin{abstract}
Os objetivos deste trabalho foram avaliar a toxicidade aguda do sulfato de cobre e do triclorfon para três espécies de Daphnia ( $D$. similis, $D$. magna e $D$. laevis) na presença ou ausência de sedimento. Os valores de $C E_{(50-48 \mathrm{~h})}$ estimados para $D$. magna foram de $0,3496 \mathrm{mg}$ de sulfato de cobre/L com sedimento e 0,0447 sem sedimento, para $D$. similis $0,2859 \mathrm{com}$ sedimento e 0,0426 sem sedimento e para $D$. laevis 0,1437 com sedimento e 0,1094 sem sedimento. A CE (50-48h) estimada para a $D$. magna foi de 299,70 $\eta \mathrm{g}$ de triclorfon/L com sedimento e 0,70 sem sedimento, para $D$. similis 381,62 com sedimento e 0,52 sem sedimento e para $D$. laevis $282,72 \mathrm{com}$ sedimento e 0,92 sem sedimento. O triclorfon mostrou-se mais tóxico que o sulfato de cobre para as três espécies estudadas, sendo que a presença de sedimento diminuiu a toxicidade de ambos para as três espécies de daphnia. Como não ocorreu diferença de sensibilidade ao triclorfon entre as três espécies na ausência de sedimento, a Daphnia laevis (espécie nativa do Brasil) pode ser usada como organismo-teste na avaliação da toxicidade aguda e crônica de inseticidas organofosforados e a base de cobre.
\end{abstract}

PALAVRAS-CHAVES: DAPHNIA; TOXICIDADE; SULFATO DE COBRE; TRICLORFON.

* $\quad$ Mestrando do Curso de Pós-Graduação em Aqüicultura de Águas Continentais, Centro de Aqüicultura (CA), Universidade Estadual Paulista (UNESP), Campus Jaboticabal (e-mail: arrolui@yahoo.es).

** Doutor em Aqüicultura de Águas Continentais, CAUNESP, Campus de Jaboticabal (e-mail: cruzcl@yahoo.com).

*** Doutor em Agronomia - Produção Vegetal, Professor do Departamento de Fitossanidade da Faculdade de Ciências Agrárias e Veterinárias, UNESP, Campus de Jaboticabal (e-mail: joaquim@fcav.unesp.br). 


\section{INTRODUÇÃO}

Os manejos inadequados, aliados ao desenvolvimento da tecnologia e de novas técnicas de criação de organismos aquáticos, muitas vezes promovem a proliferação de parasitos, pragas e doenças nos organismos cultivados (VINATEA-ARANA, 1999). A conseqüência mais grave disso envolve a forte dependência de substâncias químicas utilizadas para o controle daqueles organismos. $O$ sulfato de cobre pentaidratado $\left(\mathrm{CuSO}_{4} \cdot 5 \mathrm{H}_{2} \mathrm{O}\right)$, substância de grande aplicabilidade na aqüicultura para controle de parasitoses (como Ichthyophtirius mutifilis), pode ser empregado em enfermidades de origem bacteriana (BOYD e TUCKER, 1998). O sulfato de cobre também apresenta excelente eficiência no controle de algas e macrófitas aquáticas, pois inibe a respiração e a fotossínteses das algas (BOYD e TURKER, 1998) e elimina o fitoplâncton e as plantas aquáticas. O triclorfon (0,0-dimetil-(1-idroxi2,2,2-tricloroetil)-fosfonato), inseticida organofosforado não-sistêmico com ação de contato e ingestão, inibe a enzima acetil colinesterase (TOMLIN, 1995), estando enquadrado na classe toxicológica II (altamente tóxico).

Os inseticidas organofosforados são utilizados na aqüicultura, sem registro, para o controle de odonatas (GARADI et al. 1988), larvas de insetos (STEVENS, 1991), copépodes (CUSAK e JOHNSON, 1990 e NOGA, 1996), monogenéticos, ergasilidioses, lerneoses e argulídeos (PAVANELLI, EIRAS e TAKEMOTO, 1998), isopodes (SIEVERS et al. 1995) e monogenéticos (THONEY, 1990, PIRONET e JONES, 2000; CECCHINI e COGNETTI-VARRIALE, 2003; STEPHENS et al. 2003).

A toxicidade das substâncias químicas é afetada por fatores como solubilidade, grau de ionização, via de introdução, sítios de armazenamento, biotransformação e eliminação (LARINI, 1987). No solo e no sedimento de fundo dos espelhos d'água, o transporte, a transformação e os efeitos biológicos das moléculas orgânicas dependem muito de sua retenção pela fase organo-mineral. A biodisponilidade dos agrotóxicos nas águas depende diretamente das suas interações com os constituintes adsorventes no meio, como os sedimentos em suspensão e de fundo, pois influenciam diretamente a persistência dos compostos e seu possível resíduo (MELO e AZEVEDO, 1997). O sedimento, material particulado natural, pode ser transportado e depositado normalmente no fundo de ecossistemas aquáticos, incluindo partículas de material orgânico e água na fase intersticial de partículas (NEWMAN et al. 2000). Algumas propriedades físico-químicas da água podem afetar a taxa de adsorção das substâncias nos sedimentos, como temperatura, $\mathrm{pH}$, concentração de material em suspensão, dureza, alcalinidade, fluxo, profundidade e tamanho das partículas presentes.

A escolha de organismos-testes para realizar análises de toxicidade aquática em condições de laboratório segue os critérios utilizados pela USEPA (1985). Esses incluem as seguintes exigências: organismos representativos de grupo taxonômico ecológico, disponibilidade desses para execução de teste, existência de informações adequadas sobre a espécie, e uso (sempre que possível) de espécies nativas que sofrem o impacto (RAND e PETROCELLI, 1985). No Brasil, poucas espécies de organismos nativos são utilizadas em testes de toxicidade aguda ou crônica (FONSECA, 1991).

Os cladóceros estão entre os organismos mais utilizados para bioensaios, sendo o grupo recomendado para representar os invertebrados aquáticos (IBAMA, 1987). As daphnias são abundantes no meio aquático e exercem funções importantes na cadeia alimentar. Ocupam diferentes níveis tróficos e, quando cultivadas em laboratório, apresentam sensibilidade definida às substâncias de referência (ALMEIDA, 1987).

A Daphnia magna, muito comum no meio aquático, é utilizada na criação de alevinos de peixes (PAUW, LAUREYS e MORALES, 1981). Trata-se da espécie mais usada no mundo para teste de toxicidade devido sua sensibilidade aos agentes tóxicos, por apresentar ciclo curto e se reproduzir por partenogêneses, sendo de fácil manejo no laboratório (IBAMA, 1987; CETESB, 1991, USEPA, 1985). A Daphnia magna e a Daphnia similis são encontradas em diversos ecossistemas da Europa e dos Estados Unidos da América (SCOURFIELD e HARDING, 1966). A Daphnia laevis ocorre em diversos ecossistemas aquáticos brasileiros (ELMOR, 1997) e também em outros ecossistemas aquáticos do mundo. 
A utilização de sedimento durante a realização dos testes de toxicidade do sulfato de cobre e do triclorfon pode auxiliar no conhecimento do comportamento dessas substâncias no ambiente aquático em razão de sua capacidade de adsorção/dessorção.

O objetivo deste trabalho foi determinar a concentração efetiva $\left(\mathrm{CE}_{50-48 \mathrm{~h}}\right)$ e o efeito da presença de sedimento sobre a toxicidade aguda do sulfato de cobre e do triclorfon para três espécies de daphnia (D. magna, $D$. similis e $D$. laevis).

\section{MATERIAL E MÉTODOS}

Os experimentos foram realizados no Laboratório de Ecotoxicologia dos Agrotóxicos e Saúde Ocupacional do Departamento de Fitossanidade da Faculdade de Ciências Agrárias e Veterinárias (FCAV), Universidade Estadual Paulista (UNESP) (Campus Jaboticabal).

Utilizou-se sulfato de cobre $\left(\mathrm{CuSO}_{4} \cdot 5 \mathrm{H}_{2} \mathrm{O}\right)$ produzido pela empresa CYRQ Ind. Brasileira, grau analítico, e triclorfon (0,0-dimetil-(1-idroxi-2,2,2-tricloroetil)-fosfonato), formulação comercial denominada Dipterex $^{\circledR}$, produzido pela empresa Bayer CropScience Ltda.

\subsection{ORGANISMOS-TESTE}

As culturas de $D$. magna, $D$. similis e $D$. laevis foram conduzidas em câmara incubadora de demanda bioquímica de oxigênio (BOD) (mod. 347 FANEM), com temperatura de $20 \pm 2^{\circ} \mathrm{C}$. Os animais foram mantidos em cristalizadores de vidro de $2 \mathrm{~L}$, contendo água natural ajustada para $\mathrm{pH} 7,6$, condutividade de $160 \mu \mathrm{S} / \mathrm{cm}$ e dureza $45 \mathrm{em} \mathrm{mg/L} \mathrm{de} \mathrm{CaCO}_{3}$. Foram alimentados uma vez ao dia com suspensão de algas Scenedesmus subspicatus, concentração de $5 \times 10^{6}$ células de alga por daphnia, segundo a metodologia de cultivo do IBAMA (1987). Também receberam ração fermentada para peixe, levedura e complexo vitamínico composto por: B1 (7 mg), B2 (7 mg), B6 (5 mg), B12 (33 mg) e H (1 $\mathrm{mg}$ ) de acordo com SIPAUBA-TAVARES (2001).

\subsection{CARACTERÍSTICAS FÍSICO-QUÍMICAS DO SEDIMENTO}

Utilizou-se sedimento da camada superficial de 0 a $20 \mathrm{~cm}$ do solo de área isenta de resíduos tóxicos, localizada no Centro de Pesquisas em Sanidade Animal (CPPAR) da FCAV/UNESP (Campus de Jaboticabal). O solo foi peneirado e sua composição química e granulométrica analisada antes de ser usado nos experimentos (Tabela 1).

TABELA 1 - ANÁLISES QUÍMICAS E GRANULOMÉTRICAS DO SOLO USADO COMO SEDIMENTO NOS TESTES DE TOXICIDADE AGUDA

\begin{tabular}{lccc}
\hline Composição Química & Valores & Composição Física & Valores \\
\hline $\mathrm{pH}($ em Ca) & 5,8 & Argila $(\mathrm{g} / \mathrm{kg})$ & 440,0 \\
\hline $\mathrm{MO}\left(\mathrm{g} / \mathrm{dm}^{3}\right)$ & 24,0 & Limo $(\mathrm{g} / \mathrm{kg})$ & 226,0 \\
\hline $\mathrm{P}\left(\mathrm{g} / \mathrm{dm}^{3}\right)$ & 46,0 & Areia fina $(\mathrm{g} / \mathrm{kg})$ & 100,0 \\
\hline $\mathrm{K}\left(\mathrm{mmlcd} / \mathrm{dm}^{3}\right)$ & 4,3 & Areia grosa $(\mathrm{g} / \mathrm{kg})$ & 200,0 \\
\hline $\mathrm{Ca}\left(\mathrm{mmlcd} / \mathrm{dm}^{3}\right)$ & 47,0 & Classe textural & Argilosa \\
\hline $\mathrm{Mg}\left(\mathrm{mmlcd} / \mathrm{dm}^{3}\right)$ & 16,0 & & \\
\hline $\mathrm{H}+\mathrm{Al}\left(\mathrm{mm} / \mathrm{cd}^{3} \mathrm{dm}^{3}\right)$ & 25,0 & & \\
\hline $\mathrm{SB}\left(\mathrm{mmlcd} / \mathrm{dm}^{3}\right)$ & 67,3 & & \\
\hline $\mathrm{T}\left(\mathrm{mmlcd} / \mathrm{dm}^{3}\right)$ & 92,3 & & \\
\hline $\mathrm{V}(\%)$ & 73,0 & & \\
\hline
\end{tabular}

MO = Matéria Orgânica; H + Al = Hidrogênio + Alumínio; SB = Soma de Bases; $T$ = Capacidade de troca de cátions; V = Porcentagem de saturação de bases. 


\subsection{PROCEDIMENTO EXPERIMENTAL}

Para a realização dos experimentos foram preparadas soluções estoques da formulação comercial de triclorfon e de sulfato de cobre. A partir dessas soluções foram obtidas as soluções-testes. Em béqueres de vidro de $30 \mathrm{~mL}$ foram preparados volumes conhecidos de soluções estoques de sulfato de cobre ou de triclorfon, completando-se $9 \mathrm{~mL}$ com água natural ajustada. O volume dos béqueres foi completado para $10 \mathrm{~mL}$ com $1 \mathrm{~mL}$ de água de cultivo, contendo cinco animais neonatos.

Em outra série de béqueres de vidro de $30 \mathrm{~mL}$ foram colocados $3 \mathrm{~g}$ de sedimento e sobre esse $8 \mathrm{~mL}$ de água natural ajustada, mais os volumes conhecidos de soluções estoque de sulfato de cobre ou triclorfon, completando-se $9 \mathrm{~mL}$ com água natural ajustada. $O$ volume dos béqueres foi completado para $10 \mathrm{~mL}$ com $1 \mathrm{~mL}$ de água de cultivo, contendo cinco animais neonatos.

Foram conduzidos três experimentos compostos por 5 tratamentos, com 4 repetições e tratamento controle para cada um desses (Tabela 2). Os experimentos foram realizados em sala de bioensaios durante $48 \mathrm{~h}$, com temperatura ajustada para $20 \pm 1^{\circ} \mathrm{C}$, em ambiente escuro, sem alimentação. Após 24 e 48 h de exposição, os animais imóveis que não eram capazes de nadar durante 15 segundos foram retirados (CETESB, 1991).

\section{TABELA 2 - CONCENTRAÇÕES DE SULFATO DE COBRE E DE TRICLORFON UTILIZADAS NA DETERMINAÇÃO DA TOXICIDADE AGUDA PARA AS TRÊS ESPÉCIES DE DAPHNIA}

\begin{tabular}{|c|c|c|c|c|c|c|c|}
\hline Agrotóxico & Espécie & \multicolumn{6}{|c|}{ Concentração (mg/L) } \\
\hline \multirow{3}{*}{$\begin{array}{l}\text { Sulfato de } \\
\text { Cobre sem } \\
\text { sedimento }\end{array}$} & D. magna & 0,0 & 0,01 & 0,13 & 0,23 & 0,33 & 0,48 \\
\hline & D. similis & 0,0 & 0,01 & 0,07 & 0,14 & 0,21 & 0,28 \\
\hline & D. laevis & 0,0 & 0,01 & 0,06 & 0,12 & 0,18 & 0,24 \\
\hline \multirow{3}{*}{$\begin{array}{l}\text { Sulfato de } \\
\text { Cobre com } \\
\text { sedimento }\end{array}$} & D. magna & 0,0 & 0,1 & 0,3 & 0,5 & 0,8 & 1,0 \\
\hline & D. similis & 0,0 & 0,1 & 0,3 & 0,5 & 0,7 & 0,9 \\
\hline & D. laevis & 0,0 & 0,05 & 0,08 & 0,1 & 0,3 & 0,5 \\
\hline Agrotóxico & Espécie & \multicolumn{6}{|c|}{ Concentração ( $\eta \mathrm{g} / \mathrm{L})$} \\
\hline \multirow{3}{*}{$\begin{array}{c}\text { Triclorfon sem } \\
\text { sedimento }\end{array}$} & D. magna & 0,0 & 0,1 & 0,5 & 0,9 & 3,0 & 7,0 \\
\hline & D. similis & 0,0 & 0,2 & 0,6 & 1,0 & 4,0 & 9,0 \\
\hline & D. laevis & 0,0 & 0,2 & 0,6 & 1,0 & 5,0 & 8,0 \\
\hline \multirow{3}{*}{$\begin{array}{l}\text { Triclorfon com } \\
\text { sedimento }\end{array}$} & D. magna & 0,0 & 150 & 250 & 300 & 400 & 500 \\
\hline & D. similis & 0,0 & 250 & 300 & 350 & 450 & 600 \\
\hline & D. laevis & 0,0 & 100 & 150 & 350 & 450 & 600 \\
\hline
\end{tabular}

Estimou-se o valor da concentração efetiva $\left(\mathrm{CE}_{(50-48 h)}\right)$ de exposição ao sulfato de cobre e ao triclorfon para cada espécie de daphnia em presença e ausência de sedimento pelo método "Trimmed Sperman Karber" (HAMILTON, RUSSO e THURSTON, 1977). Para comparar a sensibilidade entre as três espécies de daphnia aos agrotóxicos, os experimentos foram analisados em delineamento inteiramente ao acaso com os tratamentos distribuídos no esquema fatorial $3 \times 2$, sendo 3 espécies de daphnia ( $D$. magna, $D$. similis e $D$. laevis) e dois sistemas de exposição (com e sem sedimento). As médias de imobilidade dos animais foram comparadas pelo teste de Tukey 5\% (SAS, 1999).

\section{RESULTADOS E DISCUSSÃO}

Os resultados da análise de variância dos valores de $\mathrm{CE}_{(50-48 h)}$ do sulfato de cobre e do triclorfon calculados para as três espécies de Daphnia (Tabela 3) evidenciaram interação significativa entre 
espécies e a presença de sedimento nos dois produtos avaliados. Os desdobramentos dos graus de liberdade dos níveis dos fatores e da interação significativa estão apresentados na Tabela 4.

TABELA 3 - ANÁLISE DE VARIÂNCIA DOS VALORES ESTIMADOS DE CE ${ }_{(50-48 H)}$ DO SULFATO DE COBRE E DO TRICLORFON PARA AS TRÊS ESPÉCIES DE DAPHNIA SEM E COM SEDIMENTO

\begin{tabular}{clc}
\hline Agrotóxico & \multicolumn{1}{c}{ Causas da Variação } & $\mathbf{F}$ \\
\hline \multirow{2}{*}{ Sulfato de Cobre } & Espécies (E) & $12,3^{* *}$ \\
& Sedimento (S) & $257,6^{\star *}$ \\
& Interação ExS & $46,6^{* *}$ \\
\hline \multirow{2}{*}{ Triclorfon } & Espécie (E) & $12,33^{\star *}$ \\
& Sedimento (S) & $1.370^{\star *}$ \\
& Interação ExS & $12,51^{* *}$ \\
\hline
\end{tabular}

**significativo ao nível de $1 \%$ de probabilidade

A toxicidade do sulfato de cobre para os organismos aquáticos depende da sensibilidade do organismo e da concentração biodisponível (OLIVEIRA-FILHO, LOPES e PAUMGARTTEN, 2004). Assim, a concentração estimada do sulfato de cobre para a $D$. magna e $D$. similis diferiu de algumas concentrações observadas na literatura. KHANGAROT e RAY (1989) calcularam para a $D$. magna $\mathrm{CE}_{(50-48 \mathrm{~h})}$ de 0,54 mg/L, utilizando Meio M4, dureza $250 \pm 15 \mathrm{mg} / \mathrm{L}$ em CaCO3. Porém, também ocorreu similaridade de concentração efetiva como outros estudos, tais como os de MOUNT e NORBERG (1984), com CE ${ }_{(50-48 \mathrm{n})}$ de $0,054 \mathrm{mg} / \mathrm{L}$ e de ELNABARAWY e ROBIDEU (1986) com $0,041 \mathrm{mg} / \mathrm{L}$.

Além dos fatores de tolerância (aclimatação), genética básica (adaptação) e regulação homeostásica das daphnias (OECD, 1996; BOSSUYT e JANSSEN, 2003), o fator sedimento exerceu influencia direta e significativa no biodisponibilidade do sulfato de cobre e do triclorfon na água para as daphnias.

TABELA 4 - DESDOBRAMENTO DOS GRAUS DE LIBERDADE DO FATOR SEDIMENTO PARA AS TRÊS ESPÉCIES DE DAPHNIA DURANTE OS TESTES DE TOXICIDADE AGUDA DO SULFATO DE COBRE E DO TRICLORFON

\begin{tabular}{lccccc}
\hline & \multicolumn{5}{c}{$\left.\mathrm{CE}_{(50-48 \mathrm{~h})} \mathrm{L}\right)$} \\
\hline Agrotóxicos (mg/L) & Sedimento & $\boldsymbol{D}$. magna & $\boldsymbol{D}$. similis & $\boldsymbol{D}$. laevis & Médias \\
\hline \multirow{2}{*}{ Sulfato de cobre } & Ausente & $0,045 \mathrm{Bb}$ & $0,042 \mathrm{Bb}$ & $0,107 \mathrm{Aa}$ & $0,065 \mathrm{~B}$ \\
\cline { 2 - 6 } & Presente & $0,347 \mathrm{Aa}$ & $0,282 \mathrm{Ab}$ & $0,140 \mathrm{Ac}$ & $0,257 \mathrm{~A}$ \\
\hline \multirow{2}{*}{ Triclorfon } & Ausente & $0,70 \mathrm{Ba}$ & $0,53 \mathrm{Ba}$ & $0,92 \mathrm{Ba}$ & $0,72 \mathrm{~B}$ \\
\cline { 2 - 6 } & Presente & $299,70 \mathrm{Ab}$ & $381,62 \mathrm{Aa}$ & $282,73 \mathrm{Ab}$ & $321,35 \mathrm{~A}$ \\
\hline
\end{tabular}

Médias seguidas por letras maiúsculas diferentes na linha indicam diferença significativa entre espécies.

Médias seguidas por letras minúsculas diferentes na coluna indicam diferença entre presença e ausência de sedimento.

Na ausência de sedimento, as Daphnia magna e Daphnia similis não apresentaram diferença de sensibilidade ao sulfato de cobre (Tabela 4) e ambas foram significativamente mais sensíveis que a Daphnia laevis. A maior tolerância de Daphnia laevis ao sulfato de cobre pode ser atribuída ao fato de tratar-se de espécie nativa e, conseqüentemente, mais rústica. VAAL et al. (1997a,b) citam que os receptores para a interação dos agrotóxicos com as biomoléculas também podem interferir na diferença de sensibilidade entre as espécies avaliadas, além de fatores como absorção, distribuição, estocagem e eliminação. 
A presença do sedimento reduziu significativamente a biodisponibilidade do sulfato de cobre na água de cultivo para $D$. magna e $D$. laevis. A adsorção crescente do sulfato de cobre ao sedimento com o passar do tempo diminuiu a biodisponibilidade na água e, conseqüentemente, resultou no aumento dos valores de $C E_{(50-48 \mathrm{~h})}$ calculados. O sedimento proporcionou diferença significativa entre os valores de $C E_{(50-48 h)}$ para as três espécies de daphnia. A maior diferença de $\mathrm{CE}{ }_{(50-48 \mathrm{~h})}$ ocorreu com a $D$. magna, cuja imobilidade do sulfato de cobre no sedimento foi 7,7 vezes maior que a concentração na água de cultivo. A segunda maior imobilidade do sulfato de cobre pelo sedimento foi verificada com a $D$. similis, 6,7 vezes maior que a concentração na água de cultivo. Por outro lado, não ocorreu diferença significativa entre os valores de $C E_{(50-48 h)}$ para $D$. laevis na presença de sedimento.

As diferenças entre os valores de $C E_{(50-48 h)}$ na ausência e presença de sedimento podem estar relacionadas com o efeito da absorção do sulfato de cobre na presença de sedimento. JONSSON e MAIA (1999) citam que na maioria dos seus estudos, o sedimento influenciou a intoxicação aguda (ou bioconcentração) de agentes químicos. Assim, a redução do efeito é causada pelo fenômeno de adsorção ao material particulado que, por sua vez, diminui a biodisponibilidade para os organismosteste.

MARTINS (1998) verificou que o aumento do teor de matéria orgânica na água diminuiu a ação tóxica do permanganato de potássio e do sulfato de cobre. Assim, a presença de sedimento nos testes pode auxiliar na estimativa real da toxicidade dos compostos de cobre para os organismos aquáticos. BURTON e LANDRUM (2003) afirmaram que a intoxicação dos organismos por substâncias químicas no ambiente aquático está relacionada com a capacidade dos compostos tóxicos de se associarem e adsorverem no sedimento. Tais características físico-químicas são as bases para a verificação de contaminação, bioacumulação e transferência dessas moléculas nos diferentes níveis tróficos.

Não se observou diferença na toxicidade aguda do triclorfon para as três espécies de daphnia estudadas na ausência do sedimento (Tabela 4). Esse resultado está de acordo com os de VESSTEEG et al. (1996), que verificaram sensibilidades semelhantes de muitos membros da família Daphnidae quanto à toxicidade aguda e crônica de diversos compostos.

Na presença de sedimento a $D$. magna e a $D$. laevis não evidenciaram diferença de sensibilidade ao triclorfon, mas ambas apresentam sensibilidade significativamente superior à $D$. similis (Tabela 4).

Foram necessárias, em média, concentrações 446,3 vezes maiores na água de cultivo para causar o mesmo efeito na $C E_{(50-48 h)}$ estimada para as três espécies de daphnia na presença do sedimento. Esse resultado demonstra o efeito da alta adsorção do triclorfon no sedimento, também observado no sulfato de cobre. Portanto, se após o contato do agrotóxico com o sedimento houver exposição de organismos-teste em diferentes períodos pode-se ter adsorção crescente dos agrotóxicos ao longo do tempo. Tal fato implicará em menor biodisponibilidade na água de cultivo e maiores valores de $C E_{(50-48 h)}$ calculados

JONSON e MAIA (1999) e BURTON e LAMDRUM (2004) citam que na maioria dos estudos, o sedimento influencia a toxicidade aguda ou a bioconcentração de agentes químicos. A redução do efeito tóxico é causada pelo fenômeno de adsorção ao material particulado, que diminui a biodisponibilidade para o organismo-teste (Tabelas 3 e 4).

Os valores de $C E_{(50-48 h)}$ calculados para as três espécies de daphnia são inferiores aos citados por TOMLIN (1995) e EXTOXNET (1996). Essas diferenças podem ser atribuídas às metodologias utilizadas e à diferença de sensibilidade dos organismos para o triclorfon.

\section{CONCLUSÃO}

O triclorfon é mais tóxico que o sulfato de cobre para as três espécies de daphnia ( $D$. magna, $D$. similis e $D$. laevias) na presença ou na ausência de sedimento. As três espécies estudadas apresentaram sensibilidades específicas ao sulfato de cobre e ao triclorfon. O sedimento diminuiu a 
biodisponibilidade do sulfato de cobre e do triclorfon na água para $D$. magna, $D$. similis e $D$. laevias. Como não ocorreu diferença de sensibilidade ao triclorfon entre as três espécies na ausência de sedimento, a espécie nativa do Brasil (Daphnia laevis) pode ser usada como organismo-teste na avaliação da toxicidade aguda e crônica dos inseticidas organofosforados e a base de cobre.

\begin{abstract}
EFFECT OF SEDIMENT PRESENCE ON THE ACUTE TOXICITY OF COPPER SULFATE AND TRICHLORFON TO THREE DAPHNIA SPECIES

The objective of the present study was to evaluate the acute toxicity of copper sulfate and trichlorfon to three Daphnia species (D. similis, D. magna and D. laevis) in the presence or absence of sediment. The estimated effective concentrations ( $\mathrm{EC}_{50-48 \mathrm{~h}}$ ) for $D$. magna were $0.3496 \mathrm{mg}$ of copper sulfate/L with sediment and 0.0447 without sediment, for $D$. similis 0.2859 with sediment and 0.0426 without sediment, and for $D$. laevis 0.1437 with sediment and 0.1094 without sediment. For trichlorfon, the $\mathrm{EC}_{(50-48 \mathrm{~h})}$ estimated for $D$. magna were $299.70 \mathrm{ng} / \mathrm{L}$ with sediment and 0.70 without sediment $\mathrm{ng} / \mathrm{L}$, for $D$. similis 381.62 with sediment and 0.52 without sediment, and for $D$. laevis 282.72 with sediment and 0.92 without sediment. Trichlorfon was more toxic than copper sulfate for the three Daphnia species studied, and the presence of sediment reduced the toxicity of these two agrochemicals. Since no difference in the sensitivity to trichlorfon in the absence of sediment was observed between the three species analyzed, D. laevis (native species of Brazil), might be used as a test organism in studies on the acute and chronic toxicity of organophosphate and copper-based insecticides.
\end{abstract}

KEY-WORDS: DAPHNIA; TOXICITY; COPPER SULFATE; TRICHLORFON.

\title{
REFERÊNCIAS
}

1 ALMEIDA, P.R. Ensaios de laboratório sobre a toxidez do DDT aos peixes guaru (Phalloceros caudimaculatus, Hensel). Arq. Inst. Biol., São Paulo, v.18 n. 2, p. 31-37, 1997.

2 BOYD, C.E; TUCKER, C.S. Pond aquaculture water quality management. Boston: Editora Kluwer Academic, 1998. 700 p.

3 BOSSUT, T.A.; JANSSEN, C.R. Acclimation of Daphnia magna to environmentally realistic copper concentrations. Comp. Biochem. Physiol. Part., C, Oxford, v. 136, p. 253-264, 2003.

4 BURTON, G.A.; LAMDRUM, P. Toxicity of sediments. In: ROCKS, G.V.; MIDDLETON, M.J.; $\mathrm{CHURCH}, \mathrm{M}$; CORIGILO, L.A. (eds). Encyclopedia of sediments and sedimentary. Dordrecht: Kluwer Academic, 2004. p. 748-751.

5 CECCHINI, S.; COGNETTI-VARRIALE, A.M. Dehydration is more effective for control of embryonic development and larval hatching of Diplectanum aequans (Monogenea, Diplectanidae) than formalin and trichlorfon. Aquacult. Intern., London, v. 11, p. 261-265, 2003.

6 CETESB. Companhia de Tecnologia de Saneamento Ambiental. Água - teste de toxicidade aguda com peixes: parte I sistema estático. In: MÉTODOS de avaliação da toxicidade de poluentes a organismos aquáticos. São Paulo, 1999, p. 1-29.

7 CUSAK, R.; JOHNSON, G. A study of dichlorvos (nuvan; 2,2, diccloroethenyl dimethyl phosphate) a therapeutic agent for the treatment of salmonids infected with sea lice (Lepeophtheirus salmonis). Aquaculture, Amsterdam, v. 90, p. 101-112, 1990.

8 EXTOXNET. Extension Toxicology Network. Pesticide information profiles: copper sulfate. Oregon: Oregon State University, 1996. p. 1-3. <http://www.extoxnet.orst.edu/pips/ coppersu.html. Acesso: 09 Abr. 2005. 
9 ELMOR, L. M. Manual de indentificação de Cladóceros Límnicos do Brasil. Brasília: Ed. Universa (UnB), 1997. 159 p.

10 ELNABARAWAY, W. T.; ROBIDEU, R.R. Relative sensitivity of daphnid specie to selected organic and inorganic chemicals. Environ. Toxicol. Chem., New York, v. 5, p 393- 398, 1986.

11 FONSECA, A. L. A biologia das espécies Daphnia laevis Ceriodaphnia silvestris (Crustácea, Cladocera) e Poecilia reticulata (Pisces, Poecilidae) o comportamento destes em testes de toxicidade aquática com efluentes industriais. São Carlos, 1991. 210 p. (Dissertação) Mestrado em Energia e Recursos Naturais, Universidade Federal de São Carlos.

12 GARADI, P.; DOMARCO, R.C.; ARAÚJO, O.J.; PINHEIRO, C.W.L. Avaliação do uso de inseticidas (orgânicos fosforados) no combate as odonatas e na seleção zooplanctonica em piscicultura de alevinagem. In: ESTUDOS de piscicultura. 2. ed. Brasília: Codevasf, 1988. $71 \mathrm{p}$.

13 IBAMA. Instituto Brasileiro do Meio Ambiente e dos Recursos Naturais Renováveis. Avaliação da toxicidade aguda para peixes. In: MANUAL de testes para avaliação de ecotoxicidade de agentes químicos. Brasília, 1987. parte D. 3.

14 HAMILTON, M.A.; RUSSO, R.C.; THURSTON, V. Trimmed Spearman-Karber method for estimating medial lethal concentrations in toxicity bioassays. Environ. Scien. Techonol., lowa, v. 7, p. 714-719, 1977.

15 JONSSON, C.; MAIA, A. Toxicidade dos herbicidas Clomazone e Quinclorac para o invertebrado aquático Daphnia similis na presença e ausência de sedimento. Pesticidas: r. ecotoxicologia e meio ambiente, Curitiba, v. 9. p.145-153, 1999.

16 KHANGAROT, B.S.; RAY, P.K. Investigation between physicochemical properties of metals and their toxicity to the water flea Daphnia magna Straus Ecotoxicol. Environ. Safety, New York, v.18 p. 109-120, 1989.

17 LARINI, L. Toxicologia. São Paulo: Editora Manole, 1987. 315 p.

18 MARTINS, L.M. Doenças infecciosas e parasitarias de peixes. 2. ed. Jaboticabal: FUNEP, 1998. p. 46-49.

19 MOUNT, D.I.; NORBERG T.J. A seven-day life-cycle cladoceran toxicity test. Environ. Toxicol. Chem., New York, v. 3, p.425-433, 1984.

20 NOGA, E.J. Fish disease: diagnosis and treatment. MOSBY: North Carolina State University, College of Veterinary Medicine, 1996. 367 p.

21 MELO, I.S.; AZEVEDO, J.L. Microbiologia ambiental. Jaguariúna: Embrapa, 1997. 440 p.

22 OECD. Organization for Economic Cooperation and Development. Daphnia sp., acute inmobilisation test and reproduction test. In: GUIDELINE for testing of chemicals. Disponível em: http://www.umwelttoxikologie.uni-konstanz.de. Acesso em: 01 Mar. de 2005.

23 OLIVEIRA-FILHO, E.C.; LOPES, R.M.; PAUMGARTTEN, F.J.R. Comparative study on the susceptibility of freshwater species to copper-based pesticides. Chemosphere, Oxford, v. 56, p. 369-374, 2004.

24 PAVANELLI, G.C.; EIRAS, J.C.; TAKEMOTO, R.M. Doenças de peixes: profilaxia, diagnóstico e tratamento. Maringá: EDUEM, 1998. 264 p. 
25 PAUW, M.; LAUREYS P.; MORALES, J. Mass cultivation of Daphnia magna Straus on ricebran. Aquaculture, Amsterdam, v. 25, p. 141-142, 1981.

26 PIRONET, F.N.; JONES, J.B. Treatments for ectoparasites and diseases in captive western Australian dhufish. Aquacult. Intern., London, v. 8, p. 349-361, 2000.

27 RAND, G.M.; PETROCELLI, S.R. Fundamentals of aquatic toxicology. Washington: Hemisphere, 1985. 665 p.

28 SAS Institute. User's Guide: statistics. Cary, 1999.

29 SCOURFIELD, D. J.; HADING, J.P. A key to the British freshwater cladocera fishnets on their ecology. $3^{\text {rd }}$ ed. London: Freshwater Biological Association Scientific, 1966. p14-15.

30 NEWMAN, M.C.; OWNBY, D.R.; MEZIN, L.C.A.; POWELL, D.C.; CHRISTENSEN, T.R.L.; LERBERG, S.B.; ANDERSON, B.A. Applying species-sensitivity distributions in ecological risk assessment: assumptions of distribution type and sufficient numbers of species. Environ. Toxicol. Chem., New York, v. 19, n. 2, p. 508-515, 2000.

31 SIEVES, G.; PALACIOS, P.; INOSTROZA, R.; DÖLZ, H. Evaluation of the toxicity of 8 insecticides in Salmo salar and the in vitro effects against the isopode parasite, Ceratothoa gaudichaudii. Aquaculture, Amsterdam v. 134, p. 9-16, 1995.

32 SIPAUBA-TAVARES L. Produção de plâncton (fitoplâncton e zooplâncton) para alimentação de organismos aquáticos. São Carlos: Editora Rima, 2001. 160 p.

33 STEVENS, M.M. Insecticide treatments used against a rice bloodworm, Chironomus tepperi (Diptera: Chironomidae): toxicity and residual effects in water. J. Econ. Entomol., Lanham,v. 84, n. 3, p. 795-800, 1991.

34 STEPHENS, F.J.; CLEARY, J.J.; JENKINS, G.; JONES, J.B.; RAIDAL, S.R.; THOMAS, J.B. Treatments to control Haliotrema abaddon in the west Australian dhufish, Glaucosoma hebraicum. Aquaculture, Amsterdam, v. 215, p. 1-10, 2003.

35 TOMLIN, C. (Ed.) The pesticide manual: a world compendium. 10 $0^{\text {th }}$ ed. Croydon: British Crop Protection Council, 1995. 1606 p.

36 THONEY, D.A. The effects of trichlorfon, praziquantel and copper sulfate on of the monogenean Benedeniella posterocolpa, a skin parasite of the cow nose ray, Rhinoptera bonasus. J. Fish. Dis., Oxford, v. 13, n. 5, p. 385-389, 1990.

37 USEPA. United States Environmental Protection Agency. Methods for measuring the acute toxicity of effluent to freshwater and marine organisms. $3^{\text {rd }}$ ed. Washington, D.C. 1985. $216 p$.

38 VAAL, M.; VAN DER WAL, J.T.; HIERMENS, J. HOEKSTRA, J. Pattern analysis of the variation in the sensitivity of aquatic species to toxicants. Chemosphere, Oxford, v. 35, p. 1291-1309, 1997a.

39 VAAL, M.; VAN DER WAL, J.T.; HOEKSTRA, J. HIERMENS, J.; Variation in the sensitivity of aquatic species in relation to the classification of environmental pollutants. Chemosphere, Oxford, v. 35, p. 1311-1327, 1997b.

40 VESSTEEG; D.J.; STALMANS, M.; DYER, S.D.; JANSSEN C. Ceriodaphnia: a comparison of their sensitivity to xenobiotics and utility as a test species. Chemosphere, Oxford, v. 34, n. 4, p. 869-892, 1996. 
41 VINATEA-ARANA, L.A. Aqüicultura e desenvolvimento sustentável: subsídios para a formulação de políticas de desenvolvimento da aqüicultura brasileira. Florianópolis: Editora UFSC, 1999. $310 \mathrm{p}$. 